\title{
1 A multiple parallel-plate avalanche counter for fission-fragment
}

\section{2 detection}

3

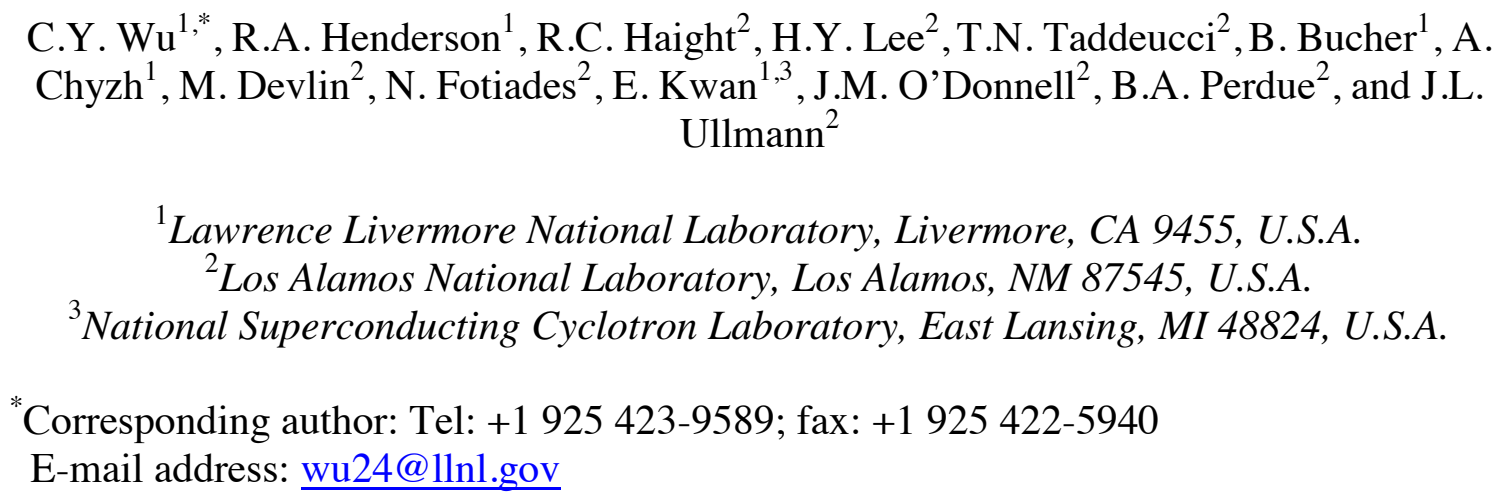

\section{Abstract}

A new low-mass multiple gas-filled parallel-plate avalanche counter for the fissionfragment detection has been developed to mark the fission occurrence in measurements of the prompt fission neutron energy spectrum as a function of incident neutron energy. It was used successfully for the neutron-induced fission of ${ }^{235} \mathrm{U}$ and ${ }^{239} \mathrm{Pu}$ with a total mass near $100 \mathrm{mg}$ each and the spontaneous fission of ${ }^{252} \mathrm{Cf}$. Both the incident neutron energy and the prompt fission neutron energy are measured by using the time-of-flight method. The design and performance of this avalanche counter are described.

\section{Keywords:}

Fission, Uranium, Plutonium, Californium-252, Parallel-plate avalanche counter 


\section{Introduction}

Two neutron detector arrays have been developed to provide the precision data on the prompt neutron emission in the neutron-induced fission of actinide nuclei as a function of incident neutron energy at the Los Alamos Neutron Science Center (LANSCE) [1,2], where neutrons are produced by bombarding a tungsten target with a pulsed $800 \mathrm{MeV}$ proton beam. One array consists of $22{ }^{6} \mathrm{Li}$-glass detectors for the detection of neutrons with the energy below $\sim 1 \mathrm{MeV}[1,2]$. The other one, consisting of 54 organic liquid scintillators, is for the detection of neutrons with energy above $600 \mathrm{keV} \mathrm{[1]} \mathrm{and} \mathrm{also} \mathrm{can}$ be used for the measurement of the prompt $\gamma$ emission in fission [3].

For the prompt fission neutron energy spectrum measurement, the energy is determined using the time-of-flight method; that is, the time difference between the fission occurrence and the detection of neutron for a given flight path. Therefore, the neutron energy resolution is determined directly by time resolutions of both neutron and fission detectors. For the fission-fragment detection, the detector requires not only a good timing response but also an ability to bear the radiation damage and intense $\alpha$-decay rate of source samples. A parallel-plate avalanche counter (PPAC) has many advantages for this application, which include fast timing, resistance to radiation damage, and tolerance of high counting rate. A PPAC also can be tuned to reduce the sensitivity to low-Z particles by lowering the bias on the anode as much as possible, which is important for experiments with $\alpha$-emitting actinides. Therefore, a PPAC is an ideal detector for experiments requiring a fast and clean trigger for fission. In the following sections, the description is given for the design and performance of a low-mass multiple PPAC for the prompt fission neutron energy spectrum measurements, which are carried out at a flight path of $21.5 \mathrm{~m}$ from the neutron source at LANSCE.

\section{Design of a low-mass multiple parallel-plate avalanche counter}

Two design principles are followed for the architecture of the current PPAC for the detection of fission fragments. One is to minimize the mass of construction material to reduce the scattering of incoming and outgoing neutrons and the other is to maximize the mass of target material to enable the measurement to be completed in a reasonable time frame with adequate statistics, while keeping the target thin enough to allow the escaped fission fragments to be detected by PPAC. The goal is to have a PPAC capable of accommodating up to $100 \mathrm{mg}$ of highly radioactive nuclei such as ${ }^{239} \mathrm{Pu}$. Shown in Fig. 1 is a schematic view of this detector system. It consists of (a) an aluminum cylindrical chamber $10 \mathrm{~cm}$ in diameter and $17.8 \mathrm{~cm}$ in length with a wall thickness of $1.59 \mathrm{~mm}$, (b) a cap to accommodate vacuum feedthroughs for transmitting 10 signals, and (c) a target assembly of 10 electrically separated PPAC modules on a common support. The thin wall of the cylindrical chamber is possible due to the use of surface-mountable gas feedthroughs, made of aluminum, instead of the standard tube fitting, for the gas flow in and out of the PPAC.

The newly designed multi-foil PPAC contains $\sim 100 \mathrm{mg}$ of target material distributed over 10 titanium foils of $3 \mu \mathrm{m}$ thickness each. The target material is electrodeposited over 
an area of $4 \mathrm{~cm}$ diameter with the surface density about $400 \mu \mathrm{g} / \mathrm{cm}^{2}$ on each side of a titanium foil using the electroplating cell described in Ref. [4]. The loaded titanium foil is then sandwiched between two $2.5 \mu \mathrm{m}$ aluminum foils, forming the cathode connected to the ground potential. Two anodes, made of the same thickness aluminum foil, are placed at either side of the cathode with a separation distance of $3 \mathrm{~mm}$. A natural Pt foil of $5 \mu \mathrm{m}$ thickness, is added to each anode to stop both $\alpha$ and fission fragments from going to the adjacent PPAC's to avoid multiple triggers for a given event. All thin foils are held by G10 fiberglass rings of $0.80 \mathrm{~mm}$ thickness. Then the assembly of the 10 individual PPAC's is inserted into a cylindrical chamber with open ends for the neutron beam entrance and exit. The open ends are sealed with $25.4 \mu \mathrm{m}$ Kapton foil. Several identical assemblies have been constructed, each with a different actinide, ${ }^{235} \mathrm{U},{ }^{239} \mathrm{Pu}$, or for calibration purposes, $\sim 2 \mu \mathrm{Ci}$ of ${ }^{252} \mathrm{Cf}$.

The detector with the multi-foil design for the fission-fragment detection has been used before in the prompt fission neutron measurements $[5,6,7]$. The major improvement here has been to reduce significantly the total foil thickness such that the attenuation to the neutron beam is less than $1 \%$ at the last foil location. In addition, 10 target foils are electrically isolated and have their own readout channels, thus the fission location can be uniquely identified. Note that the time calibration is made individually not only for each target foil to determine the energy of incident neutron but also for each neutron detector with respect to each target foil to determine the energy of outgoing neutron. We have not adopted the technique of requiring the detection of both fission fragments in coincidence as a valid fission trigger to further reduce the $\alpha$ background [8]. There are two reasons for this. First the fission and $\alpha$ events are well separated for the ${ }^{235} \mathrm{U}$ PPAC, while the fission events in the ${ }^{239} \mathrm{Pu}$ PPAC can be isolated by requiring a coincident $\gamma$ or neutron in the scintillator and subtracting the background. Second, the reduction of the trigger efficiency would be significant since the detection efficiency for one fission fragment is estimated to be $\sim 75 \%$ using the calibrated ${ }^{252} \mathrm{Cf}$ PPAC, but the detection efficiency for the partner fission fragment is degraded due to substantial energy loss after passing through the Ti foil of $3 \mu \mathrm{m}$ thickness.

\section{Operation and performance}

For stable operation of a PPAC, a stable gas pressure with continuous gas refreshing is required. This can be achieved by using a specialized gas handling system to regulate the gas flow via a feedback loop on the measured gas pressure against the preset value. Details of this gas handling system and its operation are described in Ref. [9].

The PPAC is operated typically at $\sim 4.0$ torr of isobutane with a gas flow up to $50 \mathrm{sccm}$. Two anodes in each individual PPAC are electrically connected and biased at $\sim+400 \mathrm{~V}$, producing pulse heights on the order of $1 \mathrm{mV}$ for fission fragments. Each PPAC signal is processed separately by a fast amplifier with a gain of about 600 and a bandwidth of 500 $\mathrm{MHz}$ before being sent to ZTec digitizers model 4441 with a sampling rate of $400 \mathrm{MS} / \mathrm{s}$ to extract the pulse information for each event including its time, pulse height, and three pulse integrals: (1) a short integral on the order of $20 \mathrm{~ns}$ over the peak of the pulse; (2) a longer integral over the tail of the pulse; (3) a long integral preceding the pulse for 
determining the background. The "pulse integral" referred to later in this article is the background-subtracted short pulse integral.

As mentioned earlier, the energy for incoming and outgoing neutrons is determined using the time-of-flight method. The incident neutron energy is determined by the time difference between the fission occurrence detected by PPAC and the primary proton beam pulse. An example is given in Fig. 2, where a typical spectrum of this time difference is plotted against the pulse integral of fission fragments. It is derived from the measurement of the neutron-induced fission of ${ }^{235} \mathrm{U}$, placed in the PPAC with a total mass of $112 \mathrm{mg}$. The photo-fission events, caused by prompt high-energy $\gamma$ rays produced in the neutron production target, can be seen as an isolated vertical line in Fig. 2 with the time difference of $0 \mathrm{~ns}$ relative to the prompt high-energy $\gamma$ rays and can be used in the absolute neutron energy calibration. The time resolution is better than $1.5 \mathrm{~ns}$ FWHM, which yields an energy uncertainty of better than $1 \%$ for neutron energy at $20 \mathrm{MeV}$, at the PPAC location of $21.5 \mathrm{~m}$ from the neutron production target. Two distinct peaks are visible from the projection of the Y-axis (pulse height). The lower peak near the zero pulse height is the $\alpha$ 's or the low-Z particles resulting from the possible spallation reaction occurring at the highest neutron energies. The higher peak is the fission events and has the same pulse height independent of the incident neutron energy. Despite some overlap between the fission and $\alpha$ events in the pulse height spectrum for the highest neutron energies, the fission can be identified through the entire incident neutron energy range.

For the prompt fission neutron spectrum, the energy of the outgoing neutron is determined by the time difference between the fission occurrence detected by PPAC and the neutron arrival in the neutron detector. Two types of neutron detectors are used for the current measurements; the ${ }^{6} \mathrm{Li}$-glass detector array for neutron energies below $1 \mathrm{MeV}$ $[1,2]$ and the organic liquid scintillator array for neutron energies above $600 \mathrm{keV}$ [1]. For the ${ }^{6} \mathrm{Li}$-glass detector, the neutron is uniquely identified by the known Q-value (4.78 $\mathrm{MeV})$ of the ${ }^{6} \mathrm{Li}(\mathrm{n}, \alpha)$ reaction. The full characterization of this detector array has been performed using the PPAC with $\sim 2 \mu \mathrm{Ci}$ of ${ }^{252} \mathrm{Cf}$ [2]. A typical spectrum for the time difference between PPAC and ${ }^{6} \mathrm{Li}$-glass detector vs. the pulse height measured by the ${ }^{6} \mathrm{Li}-$ glass detector is given in Fig. 3. A signal-to-noise ratio of better than 30 to 1 is observed [2]. The achieved time resolution of $\sim 5 \mathrm{~ns}$ FWHM for the ${ }^{6} \mathrm{Li}$-glass detector, determined from the time spectrum between the fission detected by PPAC and the prompt fission $\gamma$ 's detected by ${ }^{6} \mathrm{Li}$-glass detector, gives an energy resolution of about $27 \%$ for neutron energy at $300 \mathrm{keV}$ over a flight path of $30 \mathrm{~cm}$.

The organic liquid scintillator array is employed for the detection of prompt fission neutrons with energy above $600 \mathrm{keV}$. Neutrons are recognized using the pulse shape discrimination (PSD) method and their energy is determined by the time difference between the fission occurrence detected by PPAC and the neutron detected by scintillator. Shown in Fig. 4 is a typical spectrum of this time difference for the neutroninduced fission of ${ }^{239} \mathrm{Pu}$ with and without the PSD applied. The target material is placed in the PPAC with a total mass of $94 \mathrm{mg}$. The background $\gamma$ events are suppressed by nearly a factor of 4 after the PSD method is applied. This indicates that the fission event 
167 can be isolated by requiring the detection of either $\gamma$ or neutron in coincidence by the 168 scintillator. The achieved time resolution is better than 2 ns FWHM, which was 169 determined from the fission- prompt $\gamma$ coincident events and yields an uncertainty of 170 better than $9 \%$ and $30 \%$ for neutron energies at $1 \mathrm{MeV}$ and $10 \mathrm{MeV}$, respectively, over a 171 flight path of $62 \mathrm{~cm}$. The energy resolution will be improved by increasing the flight path 172 to $100 \mathrm{~cm}$ in the future measurement.

\section{Discussion}

Despite the success, two issues have been identified for this new PPAC and solutions will be implemented for the future planned measurements.

One is related to the down-scattering of outgoing neutrons due to the hydrogen content in the G-10 fiberglass rings. This issue was identified through numerical simulations of the effect on neutrons passing through the PPAC with all the material included using Monte Carlo N-Particle transport code (MCNP) [10]. The results from this simulation are shown in Fig. 5, where the improvement for the outgoing neutron spectrum with the energy below $100 \mathrm{keV}$ is demonstrated by replacing the G-10 rings with aluminum ones for the PPAC of ${ }^{252} \mathrm{Cf}$. The effect is $\sim 30 \%$ for the neutron energy at $50 \mathrm{keV}$ and about a factor of 2 for the neutron energy at $20 \mathrm{keV}$, which is consistent with our initial test results. We are replacing G-10 rings with aluminum rings to minimize the distortion of the measured spectrum. The aluminum ring has an insulated layer on one side through the anodization.

The other improvement is the effect of the field orientation on the pulse height measurement in an extreme radioactive environment such as $\sim 2 \times 10^{7} \alpha / \mathrm{s}$ in each individual PPAC with $\sim 10 \mathrm{mg}$ of ${ }^{239} \mathrm{Pu}$. By swapping the current cathode-anodes configuration to an anode-cathodes one for the existing PPAC's, the absolute number of fission events detected is increased by about $22 \%$. This may be attributed to the exhaustion of isobutane due to the intense $\alpha$ ionization in the close proximity to the target, resulting in the loss of the primary ionized electrons and leading to a change in the gas gain. With these modifications, we think this newly developed multiple PPAC is reasonably optimized for the study of the prompt neutron emission in fission.

\section{Summary}

A low-mass multiple PPAC has been successfully developed for the fission-fragment detection to study the prompt neutron emission in fission. Initial measurements for the neutron-induced fission of ${ }^{235} \mathrm{U}$ and ${ }^{239} \mathrm{Pu}$ as well as the spontaneous fission of ${ }^{252} \mathrm{Cf}$ have been fielded using this newly developed PPAC together with two neutron detector arrays for both low- and high-energy fission neutrons. These PPAC's have demonstrated excellent time characteristics and worked very well in separating fissions from $\alpha$ 's for the neutron-induced fission of ${ }^{235} \mathrm{U}$ as well as for the spontaneous fission of ${ }^{252} \mathrm{Cf}$. For the neutron-induced fission of ${ }^{239} \mathrm{Pu}$, the fission event can be isolated by requiring the detection of either $\gamma$ or neutron in coincidence by the scintillator. 
[1] R.C. Haight, H.Y. Lee, T.N. Taddeucci, J.M. O’Donnell, B.A. Perdue, N. Fotiades, M. Devlin, J.L. Ullmann, A. Laptev, T. Bredeweg, M. Jandel, R.O. Nelson, S.A. Wender, M.C. White, C.Y. Wu, E. Kwan, A. Chyzh, R. Henderson, and J. Gostic, Proc, of the International Workshop on Fast Neutron Detectors and Applications, Ein Gedi, Israel, Nov. 6 - 11, 2011 and J. Instrumentation, 7, C03028 (2012).

[2] H.Y. Lee, T.N. Taddeucci, R.C. Haight, T.A. Bredeweg, A. Chyzh, M. Devlin, N. Fotiades, J.M. Gostic, R.A. Henderson, M. Jandel, E. Kwan, A. Laptev, R.O. Nelson, J.M. O'Donnell, B.A. Perdue, S.A. Wender, and C.Y. Wu, Nucl. Instrum. Methods Phys. Res. A 703, 213 (2013).

[3] E. Kwan, C.Y. Wu, R.C. Haight, H.Y. Lee, T.A. Bredeweg, A. Chyzh, M. Devlin, N. Fortiades, J.M. Gostic, R.A. Henderson, M. Jandel, A. Laptev, R.O. Nelson, J.M. O'Donnell, B.A. Perdue, T.N. Taddeucci, J.L. Ullmann, and S.A. Wender, Nucl. Instrum. Methods Phys. Res. A 688, 55 (2012).

[4] R.A. Henderson, J.M. Gostic, J.T. Burke, S.E. Fisher, and C.Y. Wu, Nucl. Instrum. Methods Phys. Res. A 655 (2011) 66.

[5] M. Soleilhac, J. Frehaut, and J. Gauriau, J. Nucl. Energy 23, 257 (1969).

[6] S. Noda, R.C. Haight, R.O. Nelson, M. Devlin, J.M. O’Donnell, A. Chatillon, T. Granier, G. Belier, J. Taieb, T. Kawano, and P. Talou, Phys. Rev. C 83, 034604 (2011).

[7] A. Chatillon, G. Belier, T. Granier, B. Laurent, B. Morillon, J. Taieb, R.C. Haight, M. Devlin, R.O. Nelson, S. Noda, and J.M. O’Donnell, Phys. Rev. C 89, 014611 (2014).

[8] D. Tarrio et al., Nucl. Instrum. Methods Phys. Res. A 743, 79 (2014).

[9] C.Y. Wu, R. Henderson, J.M. Gostic, R.C. Haight, and H.Y. Lee, Lawrence Livermore National Laboratory, LLNL-TR-461044 (2010).

[10] T.N. Taddeucci, R.C. Haight, H.Y. Lee, D. Neudecker, J.M. O'Donnell, M.C. White, B.A. Perdue, M. Devlin, N. Fotiades, J.L. Ullmann, R.O. Nelson, T.A. Bredeweg, M.E. Rising, S.K. Sjue, S.A. Wender, C.Y. Wu, and R. Henderson, Proc. International Workshop on Nuclear Data Covariances, Santa Fe, NM, April 28- May 1, 2014, Nucl. Data Sheets 123, 135 (2015) and Los Alamos National Laboratory, LA-UR14-23460 (2014). 
256 Fig. 1 An assembly of 10 individual PPAC's is housed in a cylindrical chamber. Each PPAC, shown in the lower half, has a cathode consisting of a titanium foil with the target material marked by the white area, sandwiched between two aluminum foils as well as two anodes mounted on either side of the cathode. Each anode consists of aluminum and platinum foils and placed $3 \mathrm{~mm}$ away from the cathode. All thin foils are supported by G10 fiberglass rings except for the self-supported target foil.

Fig. 2 The pulse integral of charged particles detected by PPAC vs the time difference between PPAC and the proton beam pulse is plotted for neutron-induced reactions on ${ }^{235} \mathrm{U}$. The photoinduced fission events have no time walk, shown as an isolated vertical line with the time difference of 0 ns relative to the prompt high-energy $\gamma$ ray produced in the neutron source and can be used as the absolute calibration for the incident neutron energy.

Fig. 3 The pulse height measured by the ${ }^{6} \mathrm{Li}$-glass detector vs. the time difference between PPAC and ${ }^{6} \mathrm{Li}$-glass detector is plotted for the spontaneous fission of ${ }^{252} \mathrm{Cf}$. The neutron events are encircled by the red line, where the resonance state for the ${ }^{6} \mathrm{Li}(\mathrm{n}, \alpha)$ reaction with neutron energy at $244 \mathrm{keV}$ is visible and marked by the arrow.

Fig. 4 Shown in red is the spectrum of the time difference between the fission occurrence detected by PPAC and the prompt fission neutrons or $\gamma$ 's detected by organic liquid scintillation for the neutron-induced fission of ${ }^{239} \mathrm{Pu}$. Neutrons are identified in the scintillator using the PSD method and shown in blue. Prompt fission $\gamma$ 's detected by scintillator have no time walk and can be used as the absolute calibration of neutron energy. The locations corresponding to the neutron energies at $0.5,1.0$, and $10 \mathrm{MeV}$ are marked.

Fig. 5 The neutron energy spectra obtained by using MCNP to simulate the response to the prompt fission neutrons of ${ }^{252} \mathrm{Cf}$ detected by the ${ }^{6} \mathrm{Li}$-glass detector for a PPAC with aluminum rings (blue, 2) and a PPAC with G-10 rings (red, 3). The no-scattering spectrum for comparison is the prompt fission neutron spectrum (Maxwellian) times the ${ }^{6} \mathrm{Li}(\mathrm{n}, \alpha)$ cross section (black, 1). 


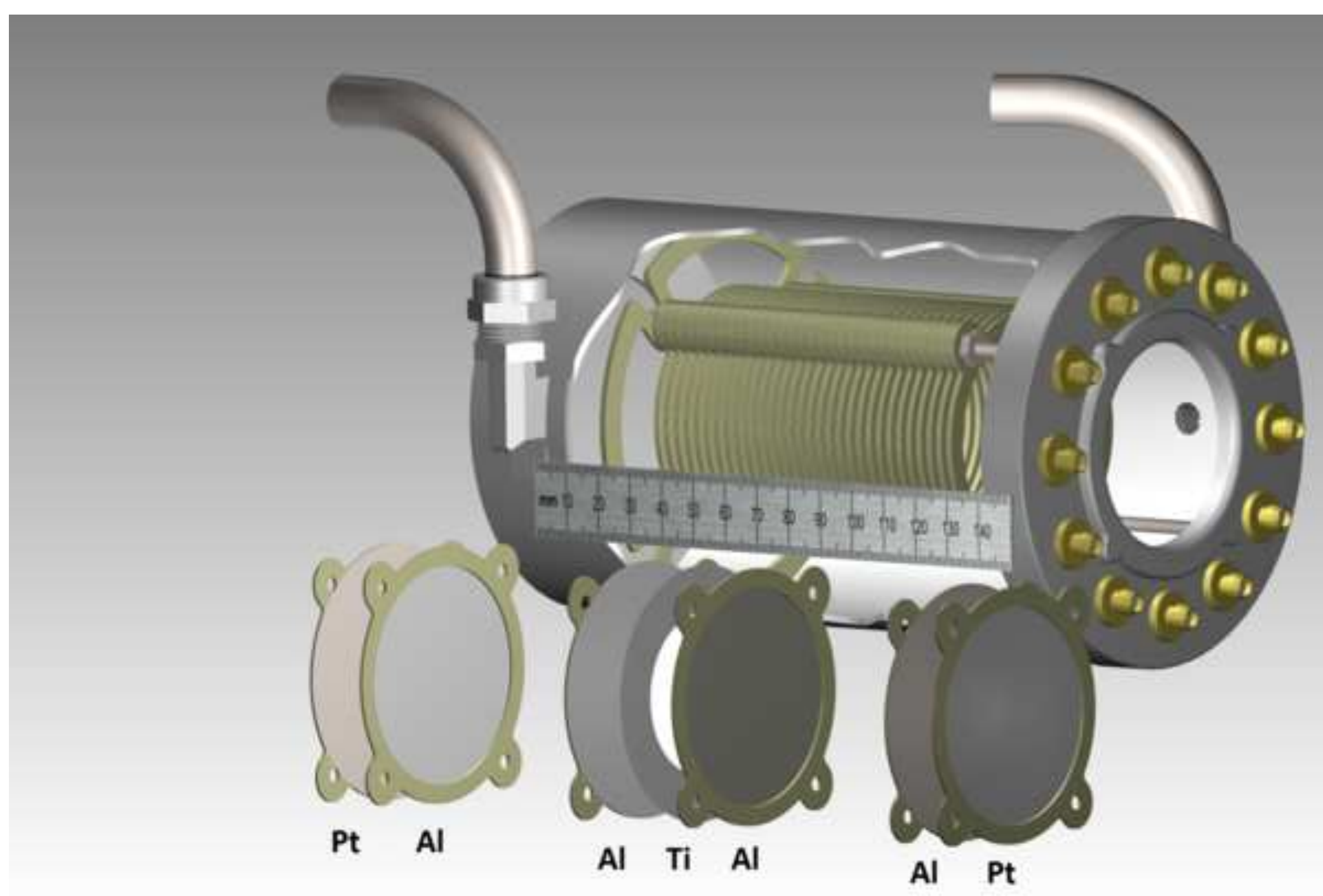




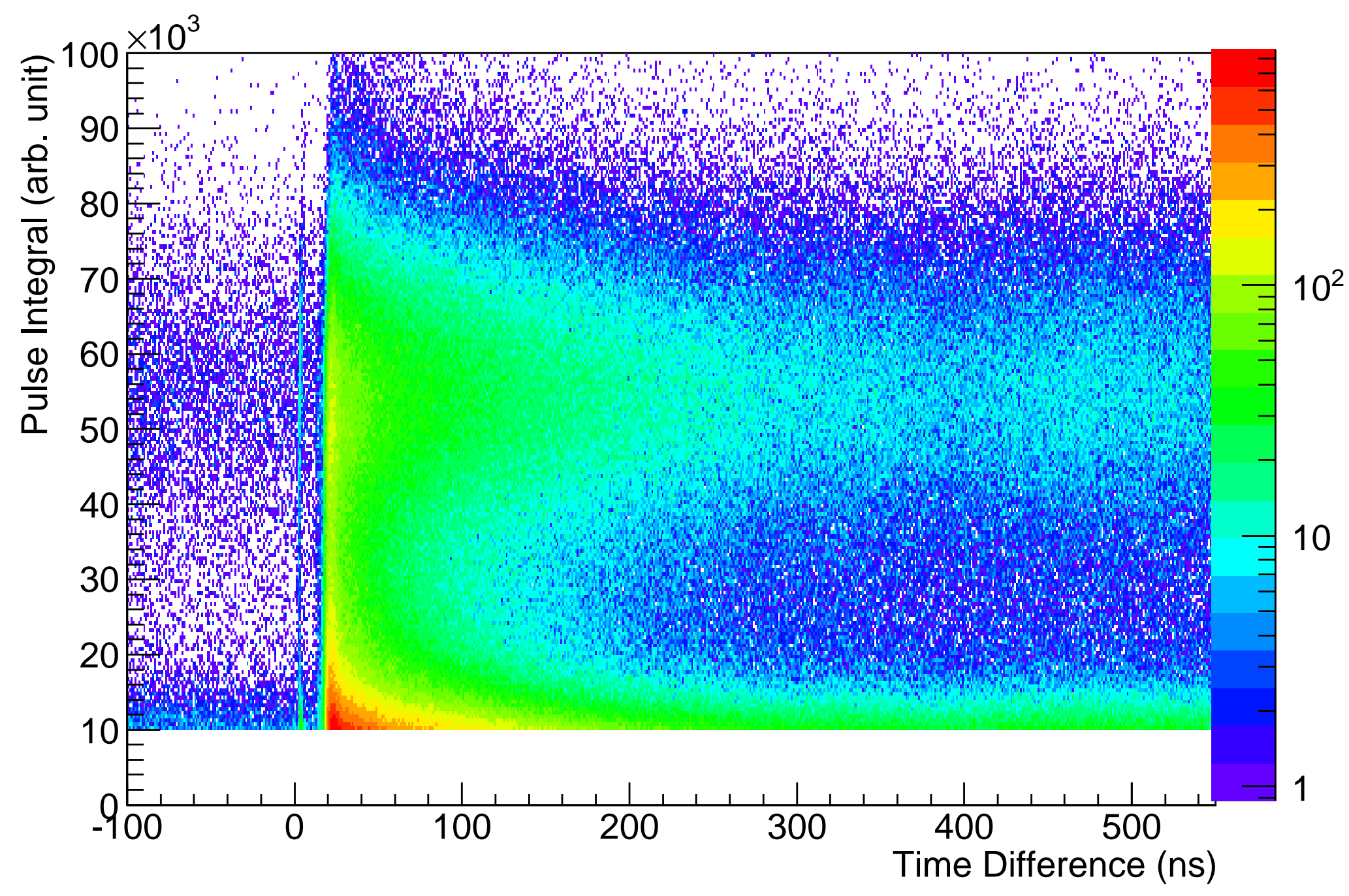




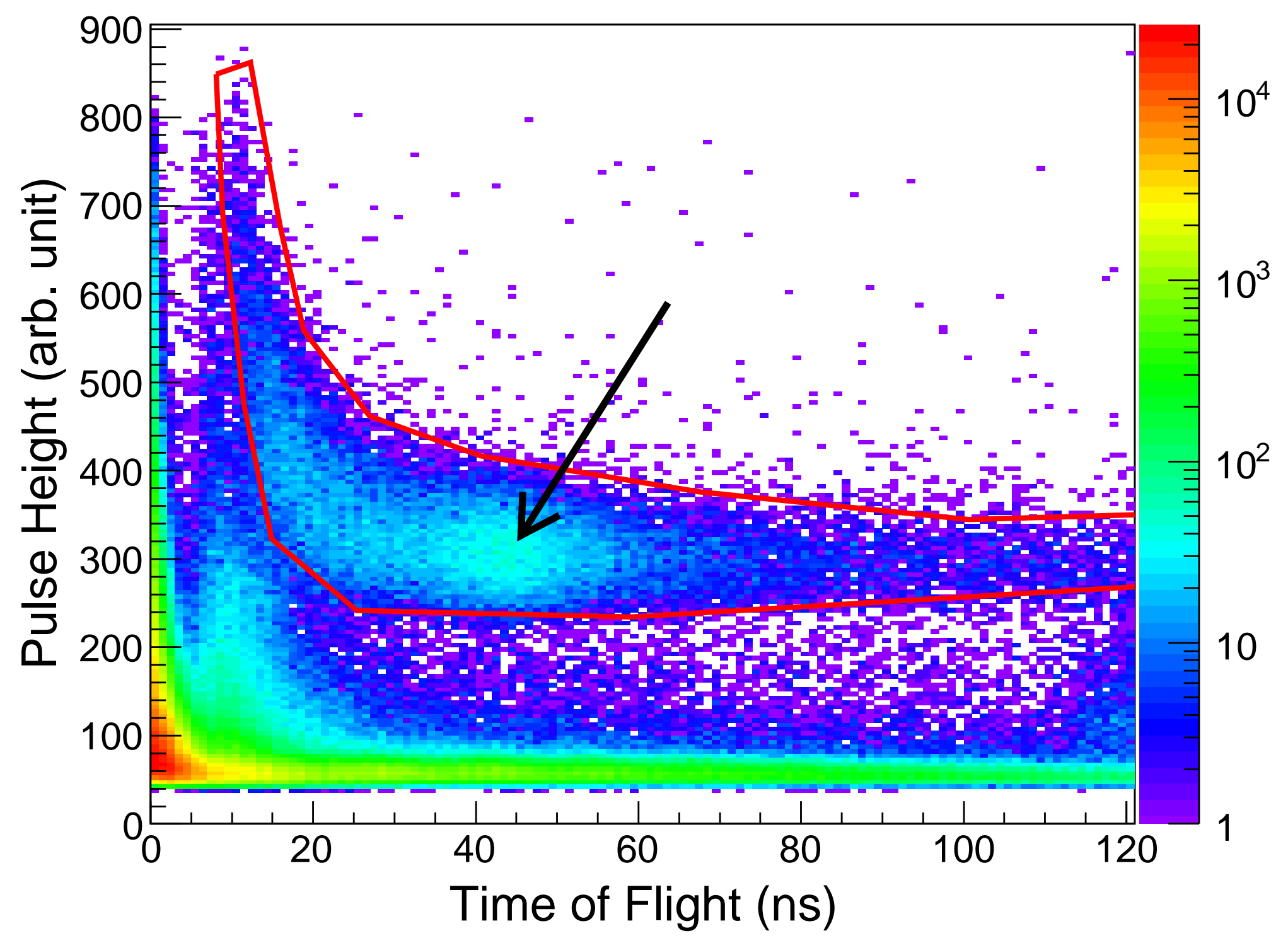




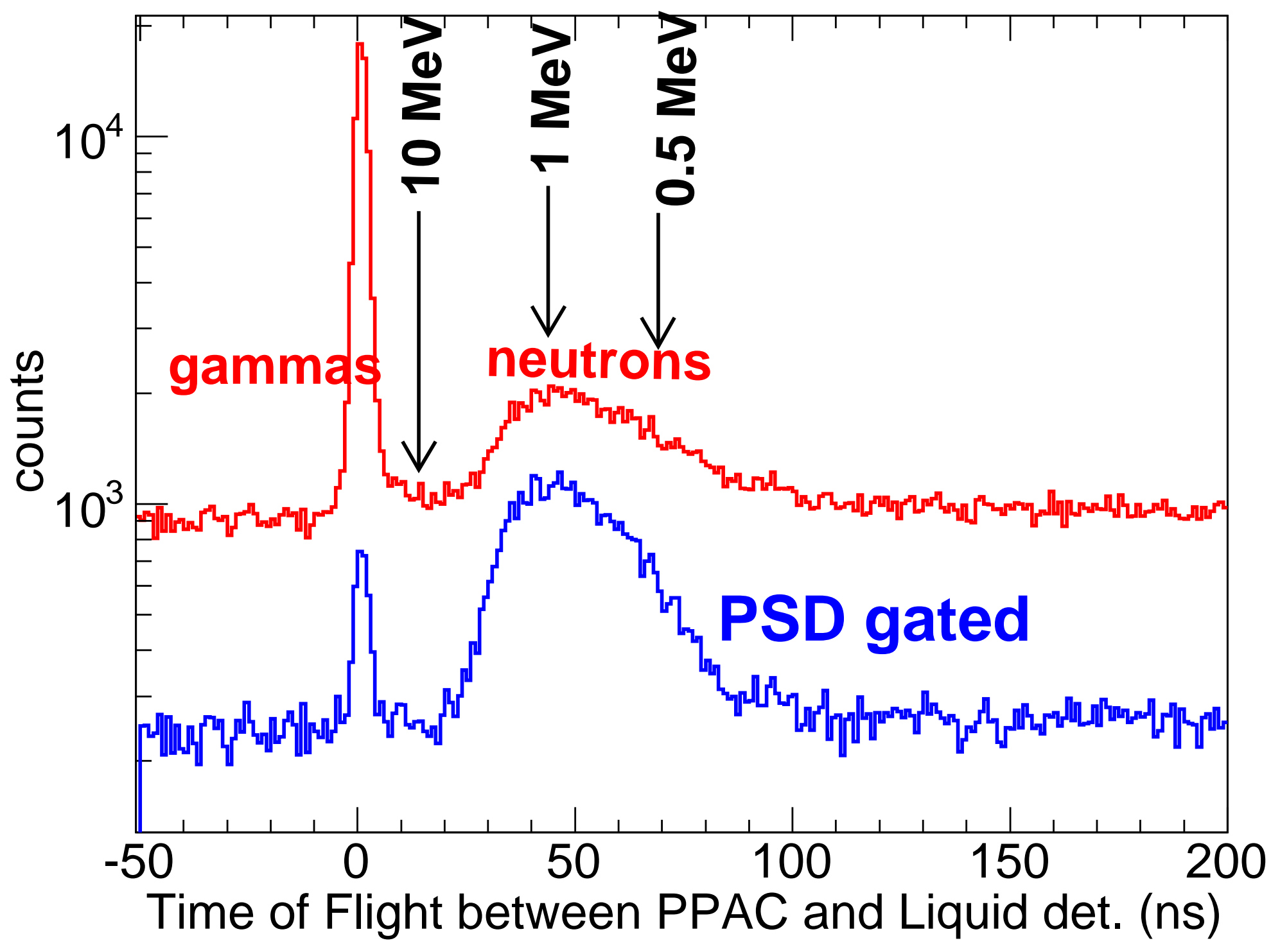




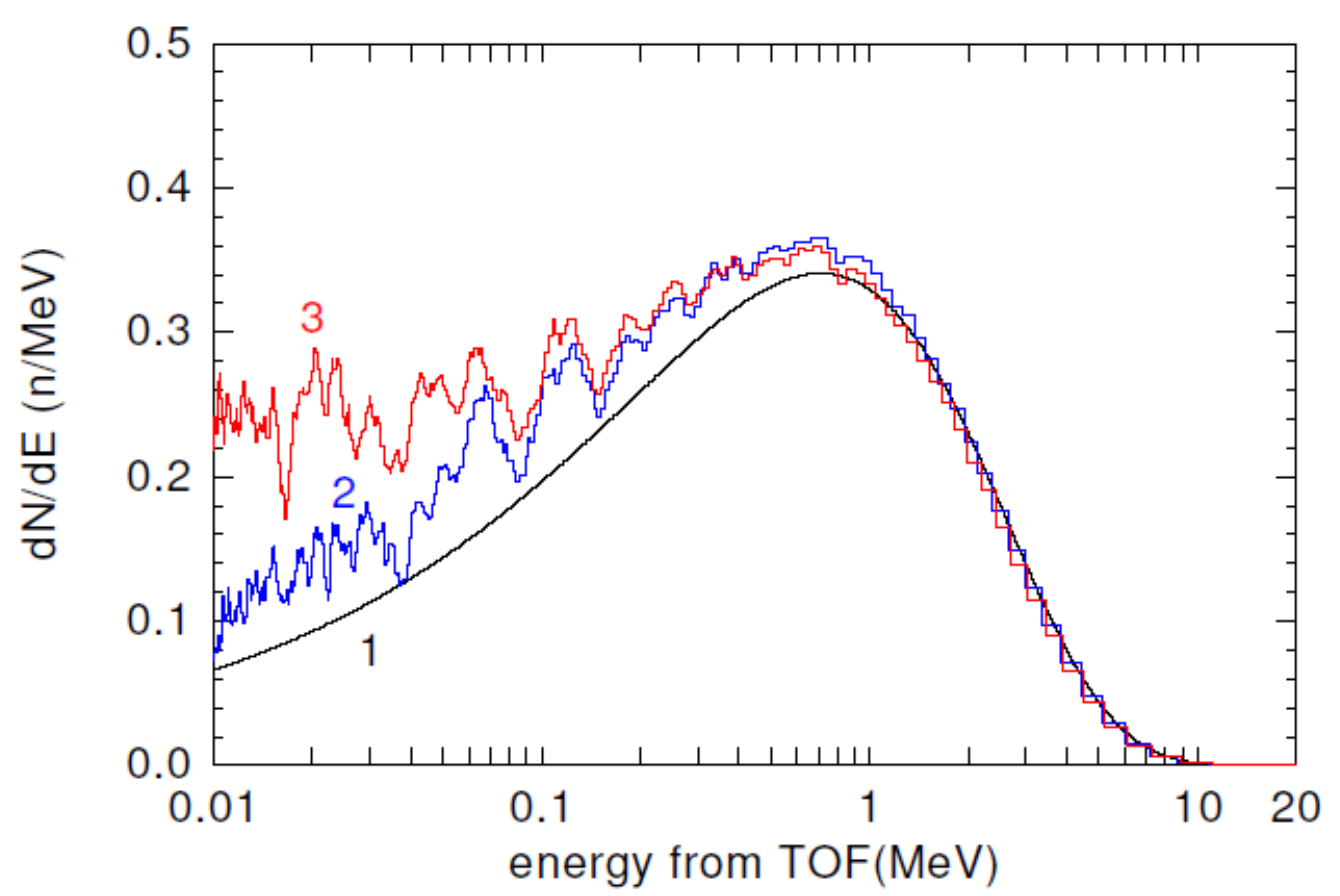

Figure 